\title{
Recurrent flares in active region NOAA 11283 ${ }^{\star}$
}

\author{
P. Romano ${ }^{1}$, F. Zuccarello ${ }^{2}$, S. L. Guglielmino ${ }^{2}$, F. Berrilli ${ }^{3}$, R. Bruno ${ }^{4}$, V. Carbone ${ }^{5}$, G. Consolini ${ }^{4}$, M. de Lauretis ${ }^{6}$, \\ D. Del Moro ${ }^{3}$, A. Elmhamdi ${ }^{7}$, I. Ermolli ${ }^{8}$, S. Fineschi ${ }^{9}$, P. Francia ${ }^{6}$, A. S. Kordi ${ }^{7}$, E. Landi Degl'Innocenti ${ }^{10}$, \\ M. Laurenza ${ }^{4}$, F. Lepreti ${ }^{5}$, M. F. Marcucci ${ }^{4}$, G. Pallocchia ${ }^{4}$, E. Pietropaolo ${ }^{6}$, M. Romoli ${ }^{10}$, A. Vecchio ${ }^{11}$, \\ M. Vellante ${ }^{6}$, and U. Villante ${ }^{6}$
}

\author{
1 INAF-Osservatorio Astrofisico di Catania, via S. Sofia 78, 95123 Catania, Italy \\ e-mail: prom@oact.inaf.it \\ 2 Dipartimento di Fisica e Astronomia - Sezione Astrofisica, Universitá di Catania, via S. Sofia 78, 95123 Catania, Italy \\ 3 Department of Physics, Universitá di Roma Tor Vergata, via della Ricerca Scientifica 1, 00133 Roma, Italy \\ ${ }^{4}$ INAF-IAPS Istituto di Astrofisica e Planetologia Spaziali, via Fosso del Cavaliere 100, 00133 Rome, Italy \\ 5 Dipartimento di Fisica, Universitá della Calabria, 87036 Rende (CS), Italy \\ ${ }^{6}$ Dipartimento di Fisica, Universitá and Area di Ricerca in Astrogeofisica, 67100 L'Aquila, Italy \\ 7 Department of Physics and Astronomy, King Saud University, 11451 Riyadh, Saudi Arabia \\ 8 INAF-Osservatorio Astronomico di Roma, via Frascati 33, 00040 Monte Porzio Catone, Italy \\ 9 INAF-Osservatorio Astronomico di Torino, Strada Osservatorio 20, Pino Torinese (TO), Italy \\ 10 Dipartimento di Fisica e Astronomia, SASS, Universitá degli Studi di Firenze, Largo E. Fermi 2, 50125 Firenze, Italy \\ 11 Istituto Nazionale di Geofisica e Vulcanologia - Sede di Cosenza, 87036 Rende (CS), Italy
}

Received 13 February 2015 / Accepted 8 August 2015

\begin{abstract}
Context. Flares and coronal mass ejections (CMEs) are solar phenomena that are not yet fully understood. Several investigations have been performed to single out their related physical parameters that can be used as indices of the magnetic complexity leading to their occurrence.

Aims. In order to shed light on the occurrence of recurrent flares and subsequent associated CMEs, we studied the active region NOAA 11283 where recurrent $\mathrm{M}$ and X GOES-class flares and CMEs occurred.

Methods. We use vector magnetograms taken by HMI/SDO to calculate the horizontal velocity fields of the photospheric magnetic structures, the shear and the dip angles of the magnetic field, the magnetic helicity flux distribution, and the Poynting fluxes across the photosphere due to the emergence and the shearing of the magnetic field.

Results. Although we do not observe consistent emerging magnetic flux through the photosphere during the observation time interval, we detected a monotonic increase of the magnetic helicity accumulated in the corona. We found that both the shear and the dip angles have high values along the main polarity inversion line (PIL) before and after all the events. We also note that before the main flare of X2.1 GOES class, the shearing motions seem to inject a more significant energy than the energy injected by the emergence of the magnetic field.

Conclusions. We conclude that the very long duration (about 4 days) of the horizontal displacement of the main photospheric magnetic structures along the PIL has a primary role in the energy release during the recurrent flares. This peculiar horizontal velocity field also contributes to the monotonic injection of magnetic helicity into the corona. This process, coupled with the high shear and dip angles along the main PIL, appears to be responsible for the consecutive events of loss of equilibrium leading to the recurrent flares and CMEs.
\end{abstract}

Key words. Sun: activity - Sun: flares - Sun: coronal mass ejections (CMEs) - Sun: magnetic fields

\section{Introduction}

Recent advances in the modelling of flares and coronal mass ejections (CMEs) have indicated that a catastrophic loss of mechanical equilibrium in the coronal magnetic configuration could be the trigger mechanism for these complex solar phenomena. Such a loss of equilibrium might drive magnetic reconnection in the magnetic field, which is stretched out by the eruption itself (Lin et al. 2003). The more complex the magnetic field configuration is, the higher the probability of a catastrophic loss of equilibrium is.

\footnotetext{
$\star$ A movie associated to Fig. 4 is available in electronic form at http://www . aanda.org
}

Several scenarios have been proposed to describe the magnetic configurations adequate to drive this dynamical instability. Various numerical experiments have demonstrated the importance of magnetic flux emergence or shear motions for driving CME-like eruptions associated with flaring activity (Shibata \& Magara 2011; Kusano et al. 2012). Moreover, in many models the flux rope is considered to be a crucial magnetic configuration. It has been shown that a flux rope can rearrange in the corona after its emergence from the convection zone, i.e., the magnetic field lines can wrap around a new central axis that is different from the original flux tube axis (Fan et al. 1998; Magara 2006).

In this regard, the so-called homologous flares are particularly interesting phenomena: the initial magnetic configuration, which was able to drive the event, is reformed after a previous 


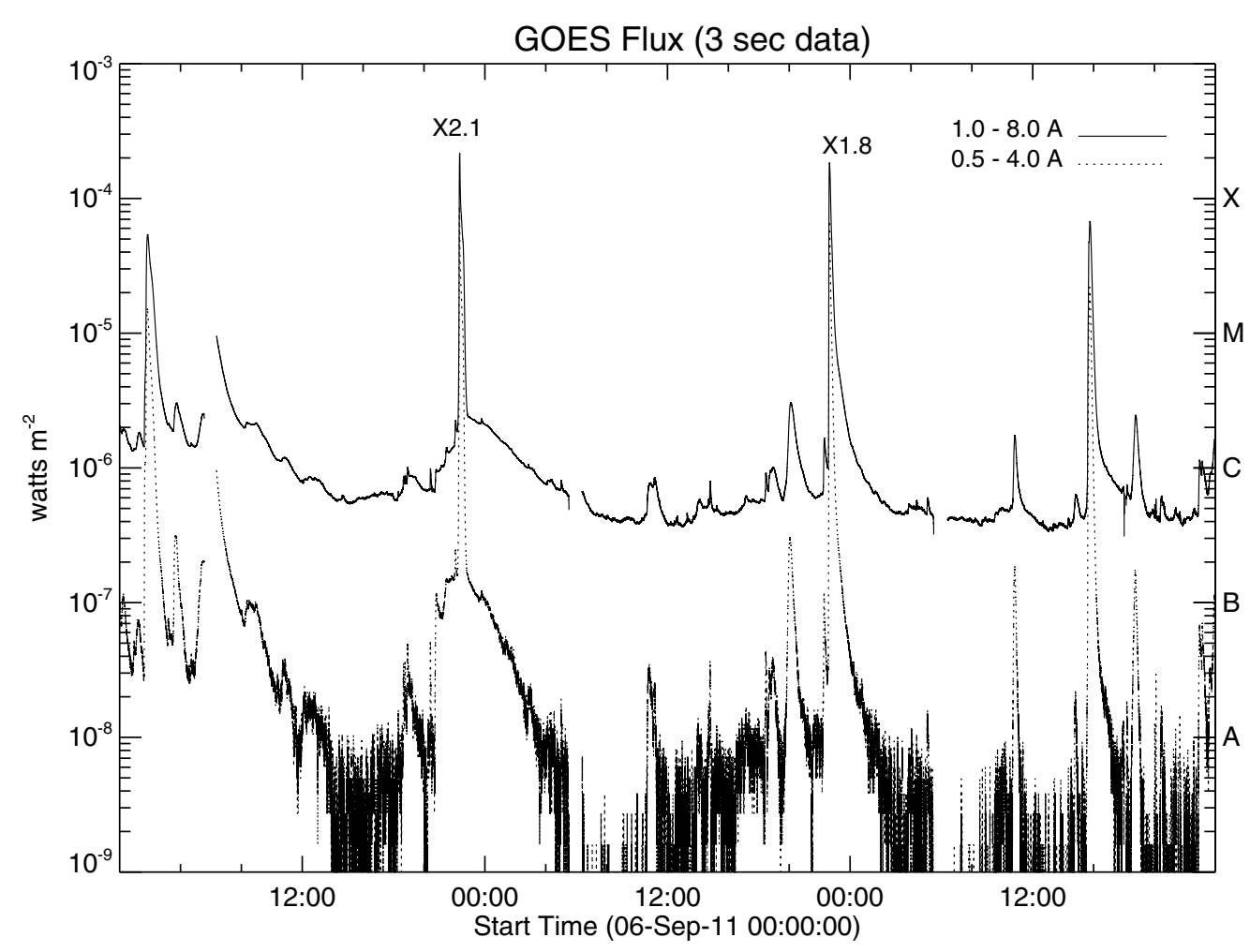

Fig. 1. GOES 1.0-8.0 ̊ (continuous line) and 0.5-4.0 ̊ (dotted line) flux light curves, for the present study's selected time interval. The most powerful X2.1 and X1.8 GOES class events that occurred during the analysed time interval are also indicated.

one. Such events pose some challenging questions about the conditions that lead to flaring, e.g., it is not clear yet whether they occur because not all of the available free magnetic energy is released, or because free energy is continuously supplied to the active region (AR). In connection with this, McClymont \& Fisher (1989) argued that new magnetic flux rising to the photosphere should carry sufficient free energy delivered by the flares, and it does not require subsequent stressing by photospheric motions.

There are several observations of homologous events; some of them were ascribed to the continuous emergence of new magnetic flux and its cancellation with pre-existing flux (e.g., Nitta \& Hudson 2001), others focused on the role of moving magnetic features in triggering the sequence of flares (e.g., Zhang \& Wang 2002).

Numerical simulations have demonstrated that eruptions can occur repeatedly both when the flux continues to emerge into the corona (e.g., Archontis et al. 2014), and when the coronal magnetic field is continuously sheared by photospheric motions (e.g., DeVore \& Antiochos 2008).

In particular, DeVore \& Antiochos (2008) in their MHD simulations showed that recurrent eruptions can be due to episodes of breakout reconnection driven by the ongoing footpoint motions. In that case the eruptions were confined, i.e., their rapidly rising, sheared field lines did not escape the Sun. The most strongly sheared field lines of the active region were quite flat prior to eruption, expanded upward sharply during the event, and lost most of their shear through reconnection with overlying flux. Lower lying field lines survive the eruption and recover their initial configuration within a few hours. This behaviour was consistent with filament disappearance followed by reformation in the same place. More recently, Archontis et al. (2014) presented the results of a 3D MHD simulation where the onset of recurrent CME-like eruptions was driven by the dynamical emergence of a horizontal twisted flux tube in a highly stratified atmosphere. They found that the eruptions were associated with the appearance of sigmoidal structures in the emerging flux region. The sequence of the recurrent eruptions is attributed to ongoing flux emergence, shearing, and reconnection in the low atmosphere. The tether-cutting reconnection (Moore et al. 2001) in the corona and the dynamical reconfiguration of the system may lead to a state that is similar to the initial one.

The present paper aims to provide a further observational contribution towards the understanding of the mechanisms that are able to reconfigure the magnetic system suitable for recurrent flares. In this context, here we present the results obtained from the measurements of the horizontal velocity fields, of the shear and the dip angles of the magnetic field, of the magnetic helicity flux, and of the energy fluxes across the photosphere in AR NOAA 11283, site of several recurrent $M$ and X GOES-class flares and CMEs. The paper is organized as follows: in Sect. 2 we briefly describe the used dataset, in Sect. 3 we report the analysis and the obtained results; and in Sect. 4 our conclusions are given and discussed.

\section{Observations}

We used the Space-weather Active Region Patches (SHARPs) data (Hoeksema et al. 2014) acquired by HMI/SDO (Schou et al. 2012) at $6173 \AA$ from September 5, 2011, at 00:00 UT to September 8, 2011, at 16:00 UT with a pixel size of 0.51 and a time cadence of $12 \mathrm{~min}$, while the AR 11283 was near the central meridian $\left( \pm 30^{\circ}\right)$.

We also used AIA/SDO (Lemen et al. 2012) images at $193 \AA$ (Fe XII; $\log T=6.1$ ) to focus on the coronal and chromospheric manifestations of the GOES M- and X-class flares. We used AIA images with a pixel size of $00^{\prime} 6$ and a time cadence of $12 \mathrm{~min}$.

As we can see from the GOES flux at 0.5-4.0 $\AA$ and at 1.0-8.0 $\AA$ (Fig. 1), four GOES M- and X-class flares occurred in the AR during the selected observation interval. The flare times 
Table 1. M- and X-class flares that occurred in AR NOAA 11283 during the observation interval, as reported by the Space Environment Center.

\begin{tabular}{ccccc}
\hline \hline Date & $\begin{array}{c}\text { Start } \\
\text { (UT) }\end{array}$ & $\begin{array}{c}\text { Peak } \\
\text { (UT) }\end{array}$ & $\begin{array}{c}\text { End } \\
\text { (UT) }\end{array}$ & $\begin{array}{c}\text { GOES } \\
\text { class }\end{array}$ \\
\hline 2011-Sept.-6 & $01: 35$ & $01: 50$ & $02: 05$ & M5.3 \\
2011-Sept.-6 & $22: 12$ & $22: 20$ & $22: 24$ & X2.1 \\
2011-Sept.-7 & $22: 32$ & $22: 38$ & $22: 44$ & X1.8 \\
2011-Sept.-8 & $15: 32$ & $15: 46$ & $15: 52$ & M6.7 \\
\hline
\end{tabular}

Notes. http://www. swpc.noaa.gov/

and classes are listed in Table 1. All these events occurred close in time with CMEs observed by LASCO/SOHO and reported in the LASCO catalogue ${ }^{1}$. We are very confident that the source of all four CMEs is AR 11283 because the running differences of AIA images at $193 \AA$ show some structures with morphology and direction of propagation very similar to the structures observed in the outer corona by the coronograph LASCO C2 (as can be seen in the available movies of the LASCO catalogue).

\section{Results}

\subsection{AR evolution}

The AR NOAA 11283 has been studied by several authors because of its strong activity when it was located near the disk centre. For instance, this privileged location allowed Jiang et al. $(2013,2014)$ to reconstruct a time sequence of static fields of the AR using a non-linear force-free field model constrained by HMI vector magnetograms. They focused on the initiation process of a X2.1 flare and a CME occurred on 2011 September 6 around 22:20 UT. They found that a flux rope was formed in the corona and grew until its axis reached a torus instability domain (Kliem \& Török 2006). The initiation of the flux rope eruption was explained by the reconnection at a coronal null point related with the field overlying the sigmoidal core. Jiang et al. (2014), following the time evolution of the magnetic field, observed the transition of an initial potential arcade to a doubleJ-shaped sheared arcade and further to an S-shaped flux rope before the eruption. The same event was studied by Feng et al. (2013), who investigated the magnetic energy partition between the flare and the CME. They argued for a similar amount of free energy for the two phenomena. The released free energy resulting from their non-linear force-free field model was about $6.4 \times 10^{31} \mathrm{erg}$. Ruan et al. (2014) showed that the filament and the overlying arcades involved in the flare were partially rooted in a sunspot which rotated at $\sim 10^{\circ} \mathrm{h}^{-1}$ during a period of $6 \mathrm{~h}$ prior to the eruption. According to their non-linear force-free field reconstruction of the coronal magnetic field, a considerable amount of magnetic energy was transported to the corona during the sunspot rotation, playing an important role in twisting and destabilizing the filament-flux rope system.

In this paper, in addition to the same X2.1 GOES class event, we also focused on all the other $\mathrm{M}$ and $\mathrm{X}$ flares/CMEs manifested while the AR was near the central meridian and, particularly, we focused on their recurrent aspect.

At the beginning of our HMI/SDO selected time interval (on 2011 September 5 at 00:00 UT) the AR was characterized by a preceding main sunspot and by several following pores, as shown in the HMI/SDO continuum image in Fig. 2a. From the comparison with the magnetogram reported in Fig. 2b, we note

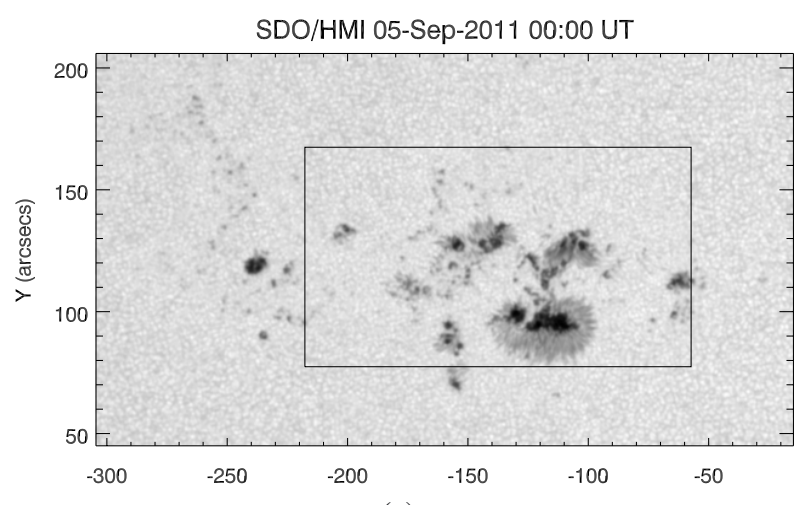

(a)

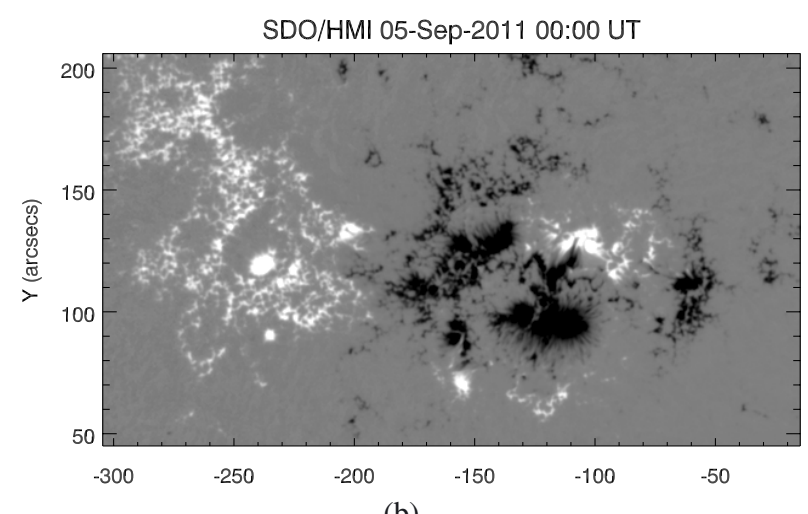

(b)
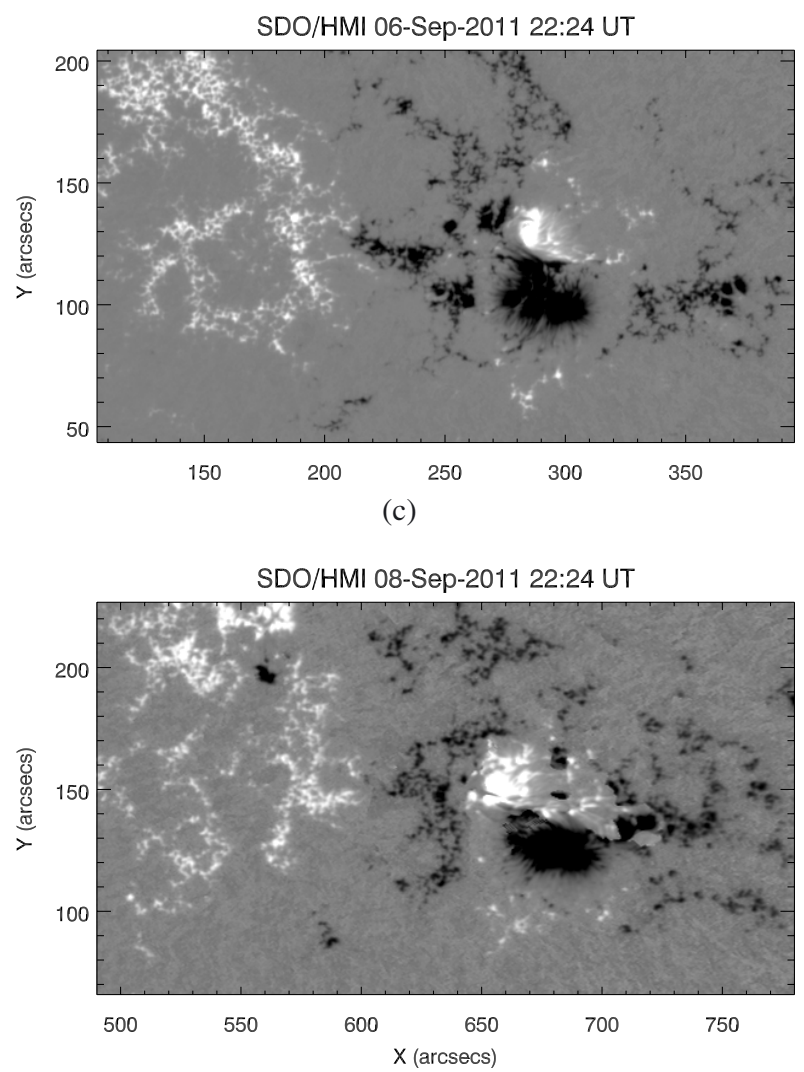

(d)

Fig. 2. a) Continuum image of AR NOAA 11283 taken by HMI/SDO on 2011 Sept. 5 at 00:00 UT. The box indicates the FOV considered in our analysis. b)-d) Sequence of vertical components of the vector magnetograms taken by HMI/SDO.

\footnotetext{
1 http://cdaw.gsfc.nasa.gov/CME_list/
} 


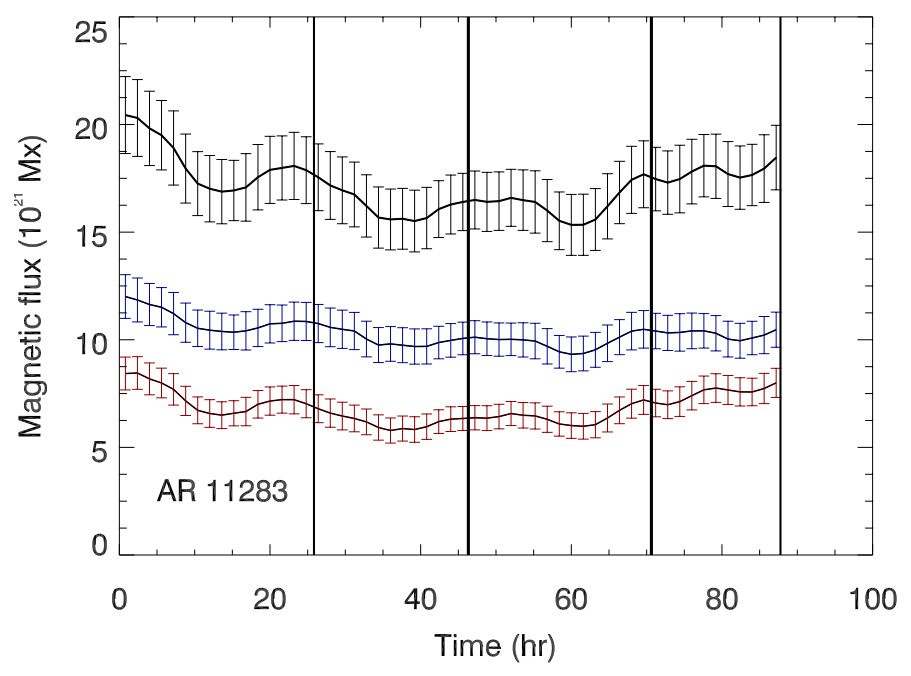

Fig. 3. Evolution of the total unsigned (black line-upper), positive (red line - lower), and negative (blue line - middle; absolute value) magnetic flux in AR 11283. $t=0$ corresponds to 2011 Sept. 5 at 00:00 UT. The shown values correspond to a 96 min time cadence. The error bars are also reported (see text). The vertical lines indicate the time when the $\mathrm{M}$ and $\mathrm{X}$ flares/CMEs listed in Table 1 occurred.

that the preceding sunspot corresponds to a more concentrated negative magnetic flux, while the more diffuse positive magnetic field was located in the eastern part of the AR. During the observation time interval the magnetic flux trend was almost constant (Fig. 3) within the errors estimated by propagating the experimental errors and considering the HMI/SDO sensitivity of $10 \mathrm{G}$ (Schou et al. 2012). However, the most interesting aspect of the AR evolution during the selected time interval is the mutual evolution of the two main magnetic polarities. In particular, the main positive feature moved eastwards while the negative one moved in the opposite direction, as we can deduce from the comparison between the magnetograms taken on 2011 September 6 and 8 (Figs. 2c and d).

During this photospheric evolution, as can be seen at $193 \AA$ (see Fig. 4 and the corresponding online movie), the AR exhibits a significant coronal activity characterized by a brightening sequence related to the above-mentioned flares, the disruption of the coronal magnetic configuration, and the reconstruction of the initial state. In particular, from the overplot of the contours of the vertical component of the photospheric magnetic field over the AIA images (Fig. 4), we note that the main activity is always located in the western part of the AR, between the two main polarities. Moreover, before the last two events, we note the continuous reformation of the S-shape EUV filament channel in the western part of the AR where the flares take place (see the white arrows in Figs. $4 \mathrm{c}$ and d).

\subsection{Horizontal velocity fields}

Following the method described by Schuck (2008), we compared the magnetic field between two magnetograms taken with a time interval of $24 \mathrm{~min}$ in order to derive the horizontal velocity fields by means of the Differential Affine Velocity Estimator method for vector magnetograms (DAVE4VM; Schuck 2008). We used a full width at half maximum of the apodization window of 11 pixels $\left(5^{\prime \prime} .5\right)$.

In Fig. 5 we show the horizontal velocity maps taken before each of the event listed in Table 1. Along the main PIL, we

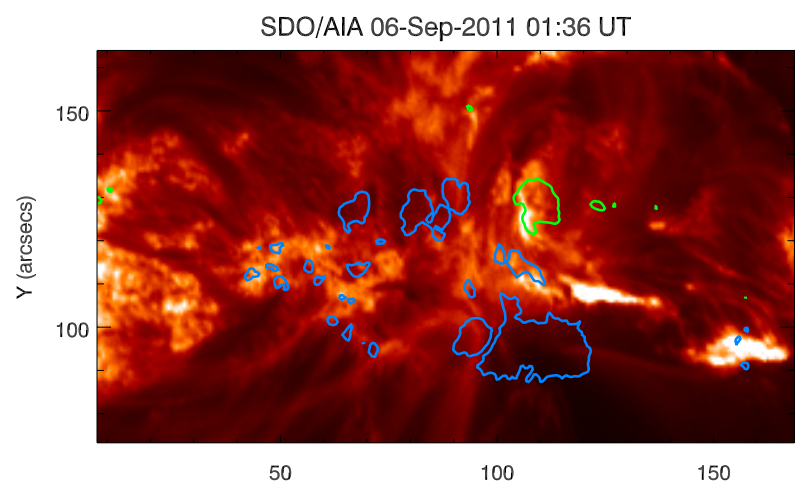

(a)

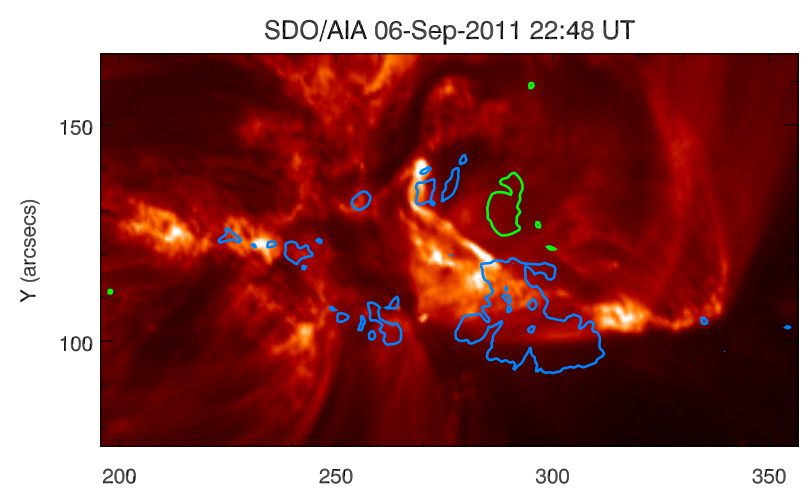

(b)

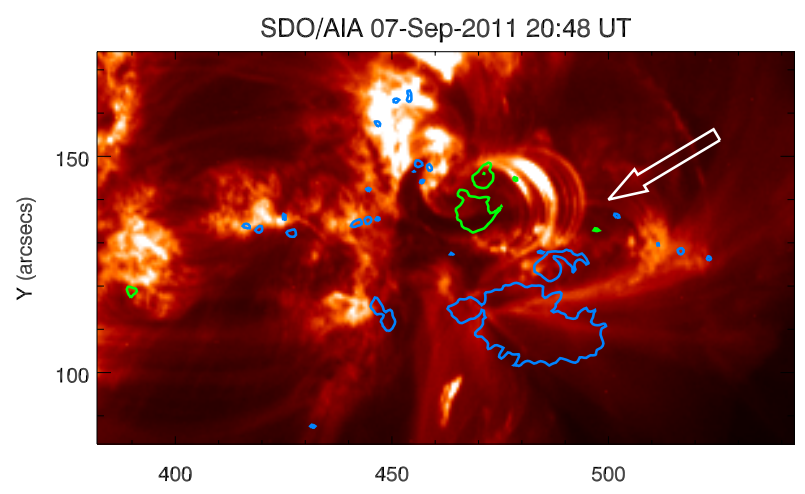

(c)

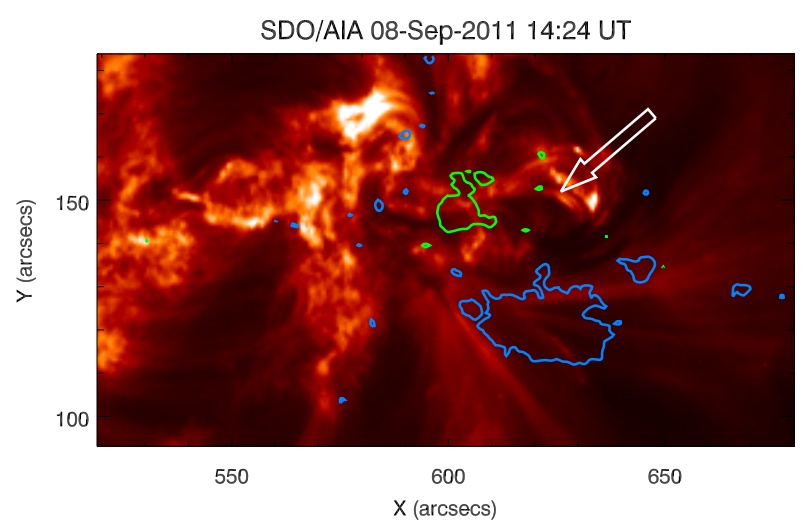

(d)

Fig. 4. Sequence of $193 \AA$ (Fe XII; $\log T=6.1$ ) images taken by AIA/SDO a few minutes before each of the events listed in Table 1. The green and blue contours correspond to the vertical components of the photospheric magnetic field equal to $+1000 \mathrm{G}$ and $-1000 \mathrm{G}$, respectively. The full temporal evolution is shown in the online movie. 


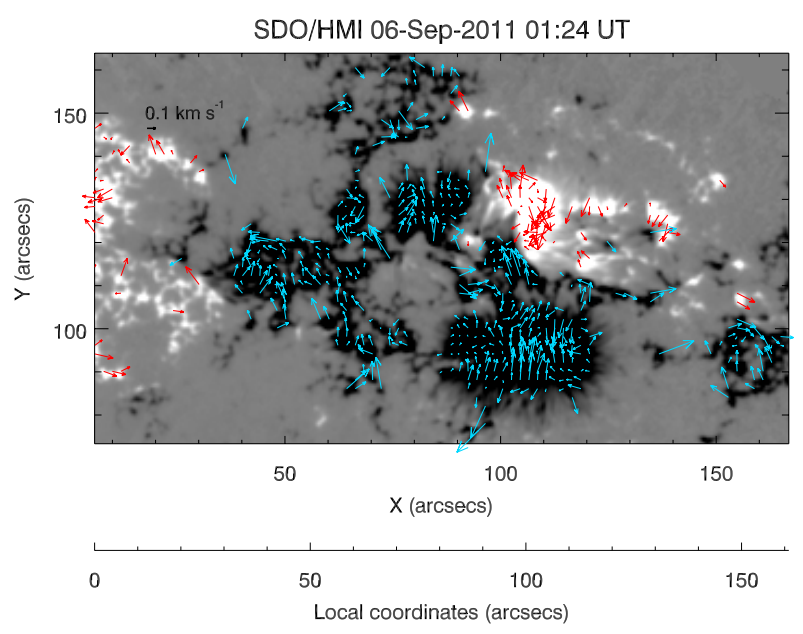

(a)

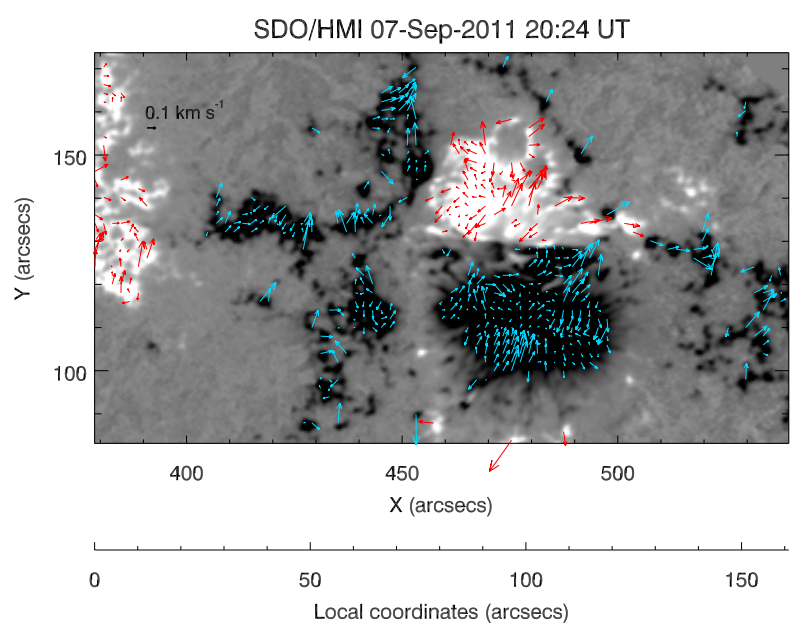

(c)

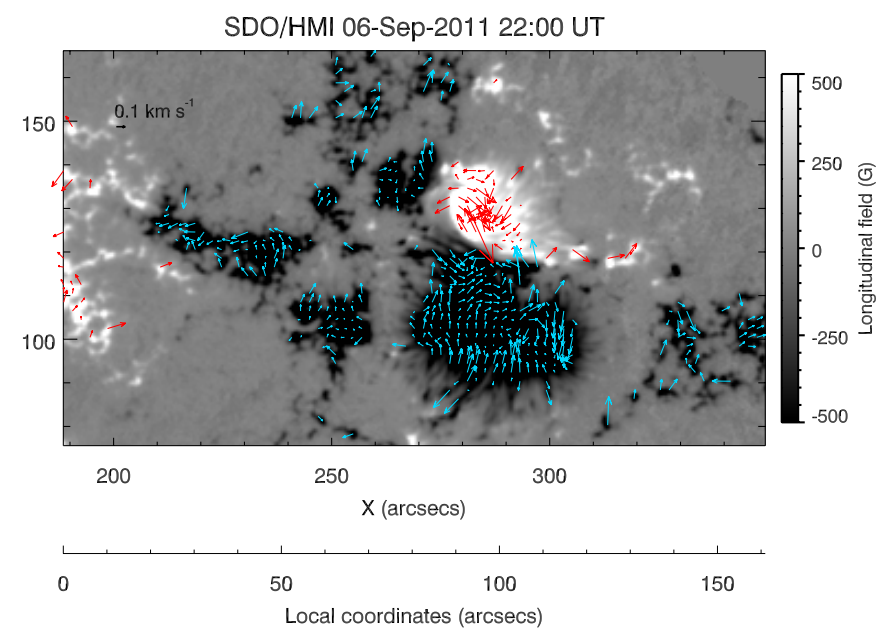

(b)

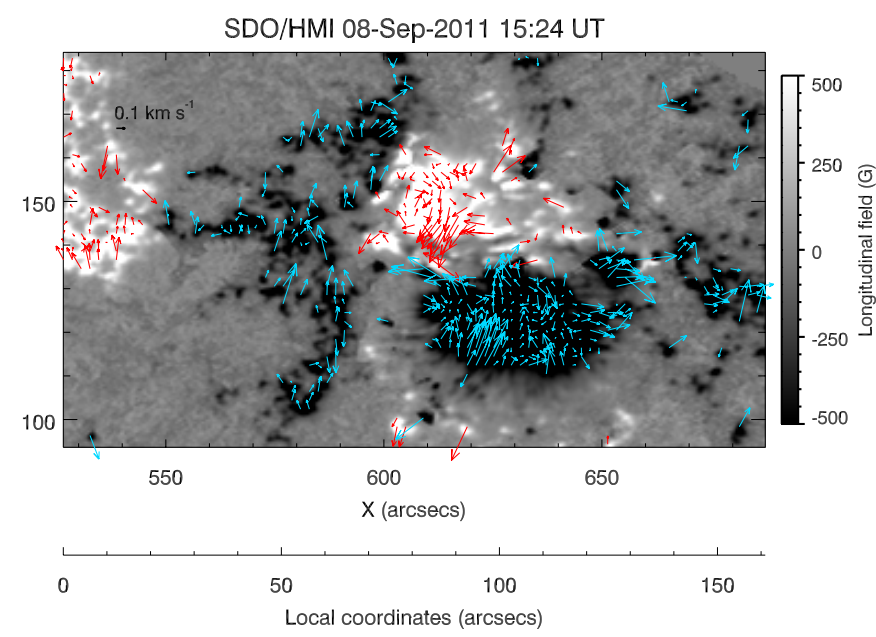

(d)

Fig. 5. Sequence of horizontal velocity maps of AR NOAA 11283 derived from HMI/SDO data taken a few minutes before the flares listed in Table 1.

note the persistent displacements of the positive and negative polarities eastwards and westwards, respectively, during the whole observation time interval. In fact, at the beginning of our observation time interval, the barycentres of both polarities have approximately the same longitudes (Fig. 5a), while at 15:24 UT on 2011 September 8 (Fig. 5d) their longitudes differ by about $15^{\prime \prime}$. Moreover, in the same maps it is also possible to recognize the clockwise rotation of the positive spot, already interpreted by Ruan et al. (2014) as the main mechanism able to transport a considerable amount of magnetic energy into the corona before the X2.1 flare occurred on September 6 .

\subsection{Shear and dip angles}

We also evaluated the shear between the observed (measured) horizontal field and the horizontal field derived through a potential field extrapolation (Wang et al. 1994), computed using the method described by Alissandrakis (1981). We estimated the shear angle following the procedure of Falconer et al. (2002) and Jiang et al. (2014). In addition, we computed the dip angle, which measures the difference between the inclination angle of the observed field and that of the potential field (see, e.g., Gosain \& Venkatakrishnan 2010; Petrie 2012).

In Figs. 6-9 we show the maps of the shear angles (left columns) and of the dip angles (right columns) report for each of the four flares, deduced from the vector magnetograms taken before and after the $\mathrm{M}$ and $\mathrm{X}$ flares. In the bottom panels of the same figures we also show the difference maps obtained from the above mentioned maps. We note that the shear reaches the highest value of the order of $100^{\circ}$ near the main PIL and that this value decreases after the occurrence of all the coronal events. The dip angle reaches its highest values around the rotating positive sunspot. From the difference maps we note that the estimated values of both angles decrease significantly only after the onset the two X-class flares. Therefore, it seems that the more intense the flare, the greater the dip and the shear variations.

\subsection{Magnetic helicity flux distribution}

The continuous motion towards opposite directions of the main positive and negative polarities and the resulting shear of the magnetic field lines connecting the two polarities probably 


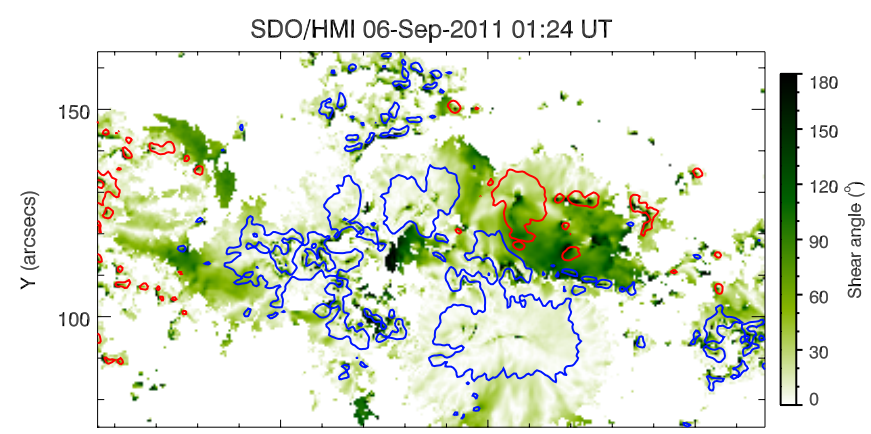

(a)

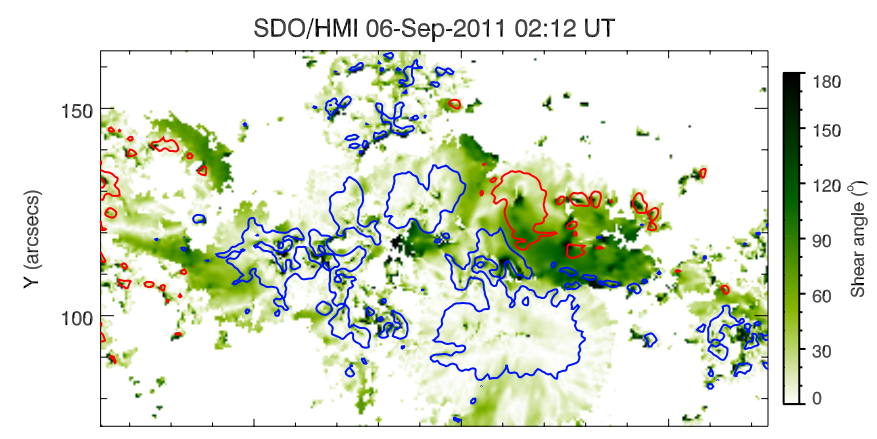

(c)

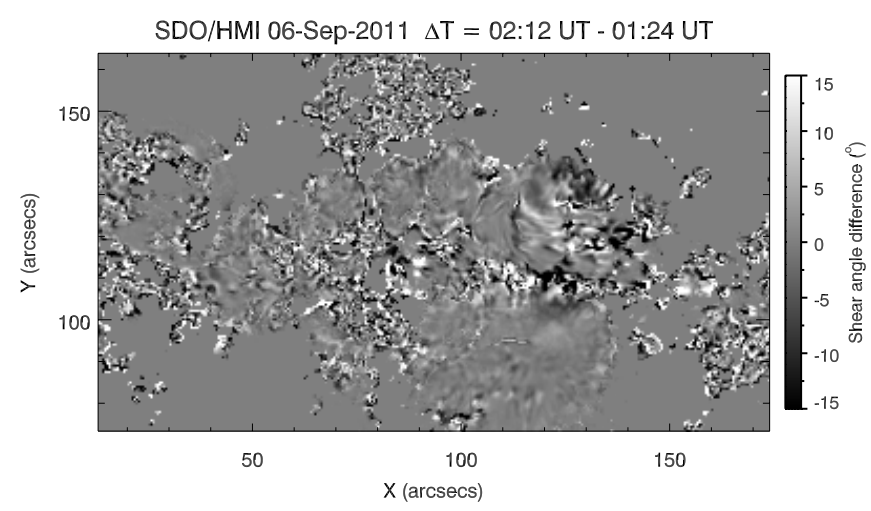

(e)

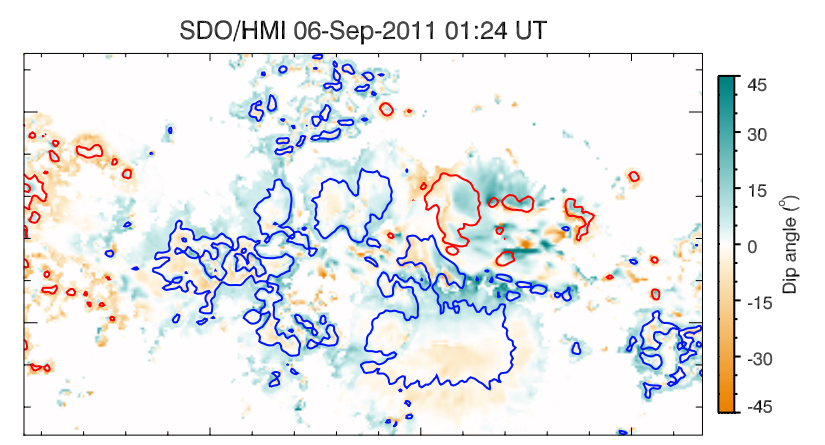

(b)

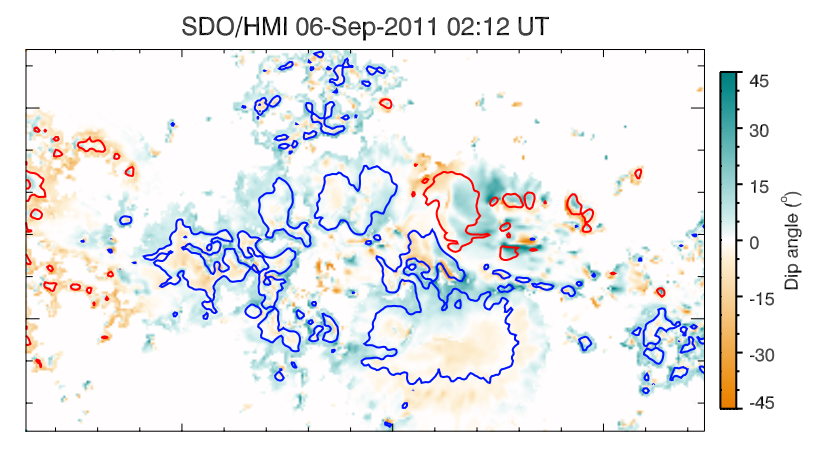

(d)

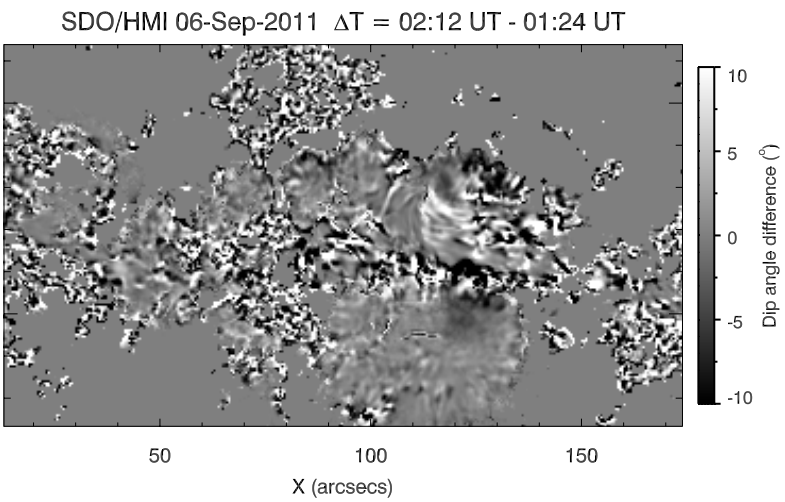

(f)

Fig. 6. Left column: shear angle maps of AR NOAA 11283 derived from HMI/SDO data taken before a) and after b) the first flare listed in Table 1 and a map obtained from the difference between them c). Right column: same as left column, but for the dip angle.

played an important role in the storage of magnetic energy released during the subsequent events, as already reported by Jiang et al. (2013, 2014). Therefore, we also paid particular attention to the distribution of the magnetic helicity in this area of the AR, as this quantity usually provides additional information for a better understanding of the onset mechanism of flares and CMEs.

We note that the magnetic helicity is an appropriate useful physical quantity that provides a view of the complexity of the magnetic field configuration. The direct computation of magnetic helicity in an AR requires the knowledge of the magnetic field connectivity in the entire volume under study, but since magnetic field measurements are mainly taken at the photospheric level, the most common way to estimate magnetic helicity is through the computation of the magnetic helicity flux from the convection zone $(\mathrm{d} H / \mathrm{d} t)$. This is possible for example by using the measurements of the vertical component of the magnetic field and the computation of the displacements of the photospheric magnetic structures, as in the approach proposed by Pariat et al. (2005). For the purposes of our present investigation, we estimated the magnetic helicity flux adopting the above-mentioned method and then we computed the magnetic helicity accumulation in the AR since the beginning of the observations (see Fig. 10). We note that the AR shows a prevalent positive magnetic helicity accumulation during the observing time interval, although it is located in the northern hemisphere, i.e., it does not obey the general cycle-invariant hemispheric helicity rule (Liu et al. 2014). It is also meaningful that the monotonic trend of the magnetic helicity accumulation in the corona is not supported by magnetic flux emergence (compare with Fig. 3), but probably by the peculiar horizontal velocity field. 


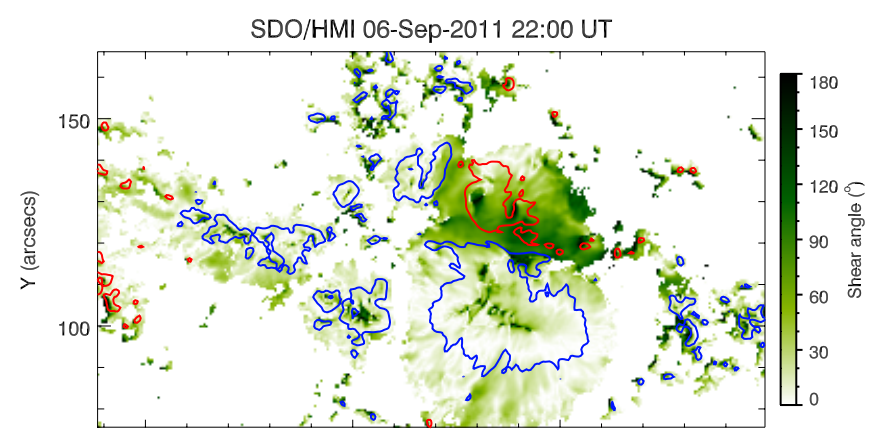

(a)

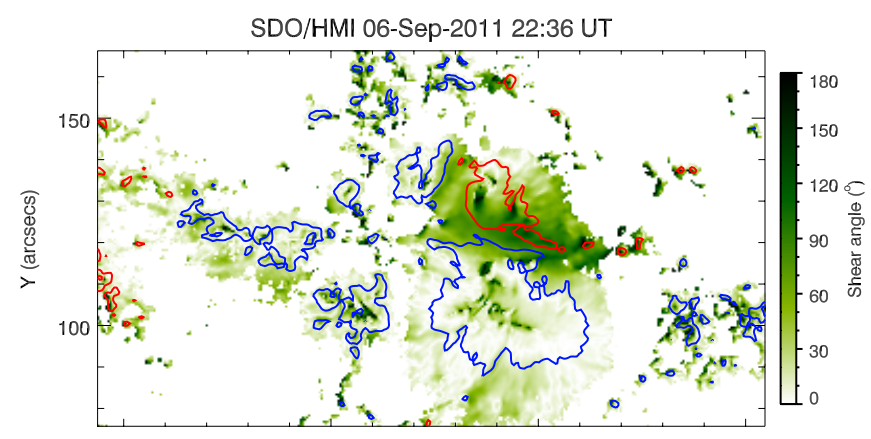

(c)

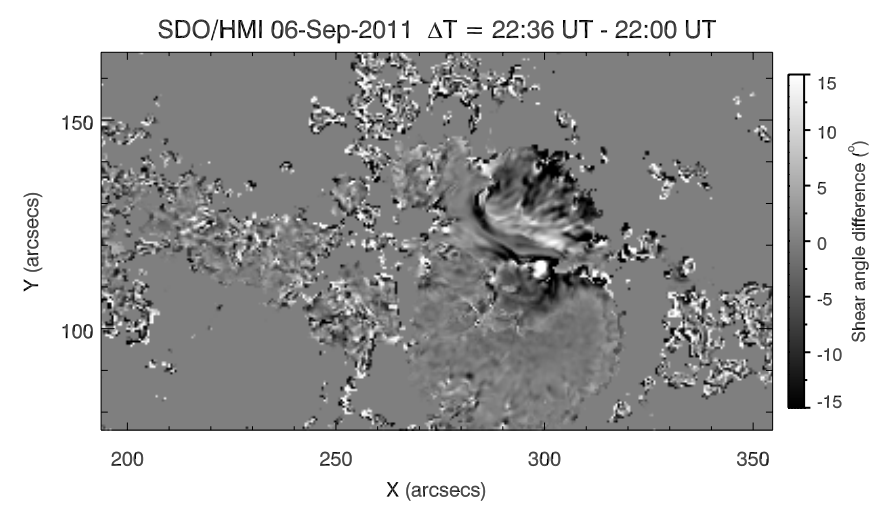

(e)

Fig. 7. Same as Fig. 6, but for the second flare listed in Table 1.
In Fig. 11 we show an example of the magnetic helicity flux map deduced by applying the method of Pariat et al. (2005). As usual, the flux map appears not uniform but fragmented in different regions of opposite sign and not corresponding to the distribution of the magnetic field in the photosphere. Actually, this aspect can be ascribed in part to the limits of the applied method and in part to the real helicity flux distribution. In fact, although the Pariat et al. (2005) method reduces the presence of spurious signals corresponding to fake polarities in comparison with previous methods (Chae 2001), we cannot exclude the possiblity that the helicity flux fragmentation comes from this noise. However, Romano \& Zuccarello (2011) recently analysed the temporal variation of the maps of magnetic helicity flux by measuring the fragmentation of the patches, determined by grouping unipolar, contiguous pixels with a helicity flux density greater than $5 \times 10^{17} \mathrm{Mx}^{2} \mathrm{~cm}^{-2} \mathrm{~s}^{-1}$ in absolute value. In particular, they

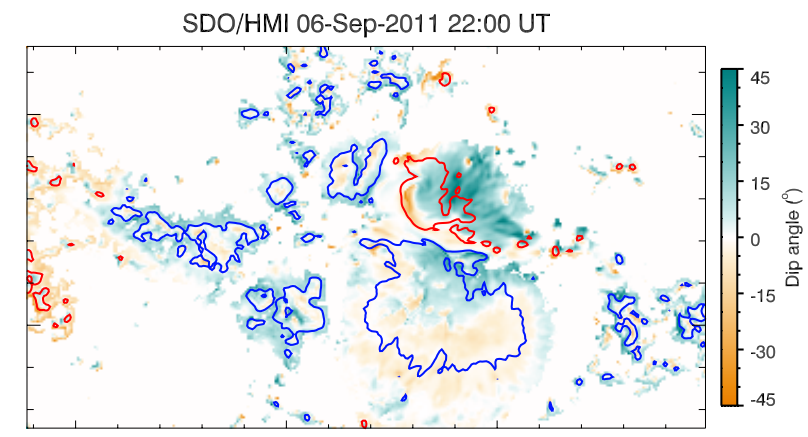

(b)

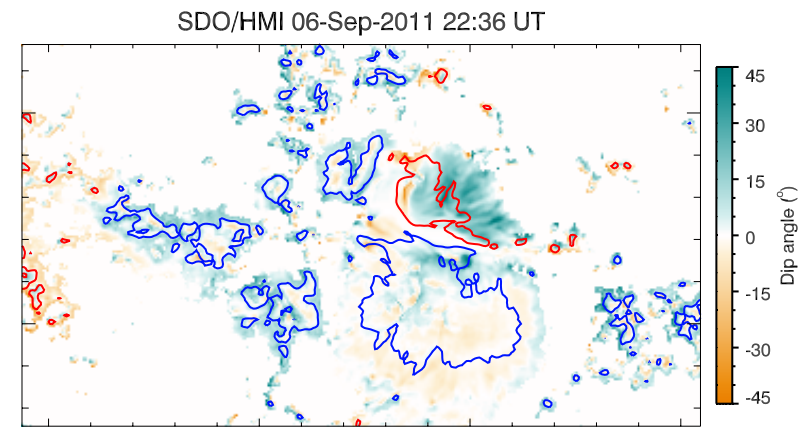

(d)

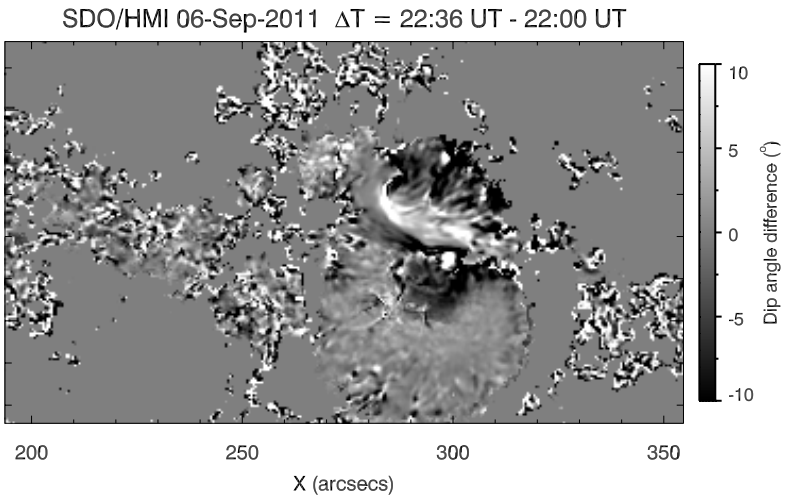

(f) studied the temporal correlation between the number of these patches and the flare and CME occurrence, finding that the fragmentation of the patches provides a useful indication of the evolution of AR complexity. The more fragmented the maps of the magnetic helicity flux are, the higher the flare and CME frequency are. Most of the eruptive events occurred for low values of the difference of the number of patches with opposite signs of magnetic helicity flux.

In our case, during the whole observation time interval, the main contribution to the positive helicity flux is provided by the main positive and negative photospheric magnetic structures that move eastwards and westwards, respectively. This indicates that the horizontal velocities are able to inject new positive magnetic helicity in the corona independently from the flare/CME activity of the AR. Moreover, we note the presence of magnetic helicity flux concentrations of opposite polarity along the PIL. This 


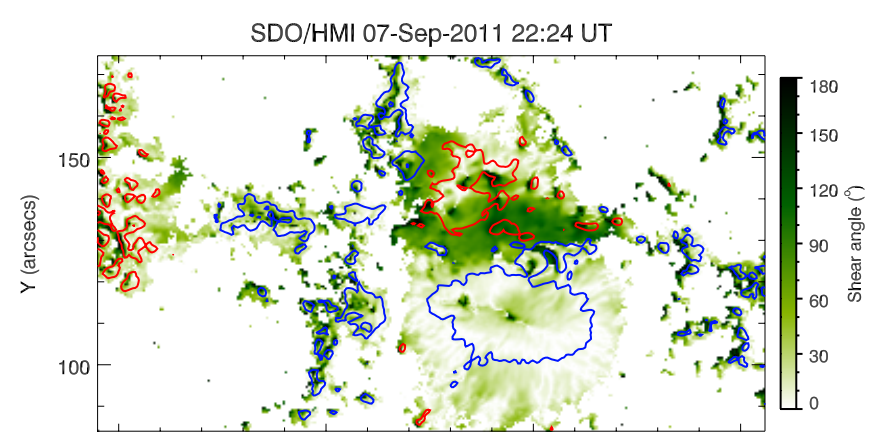

(a)

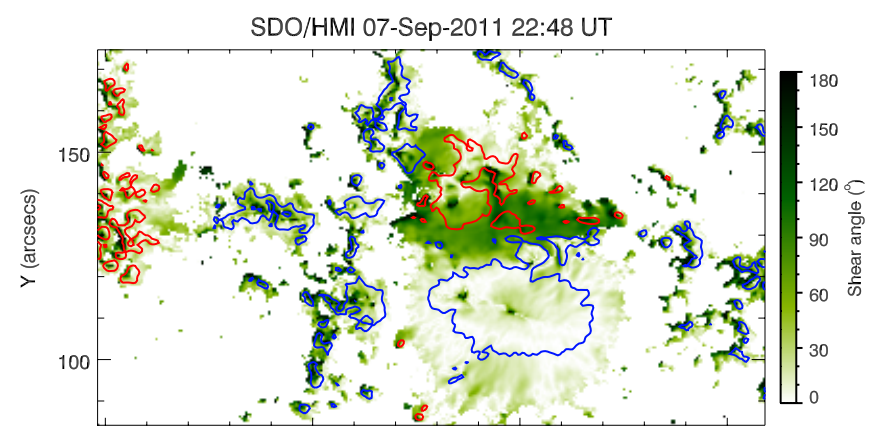

(c)

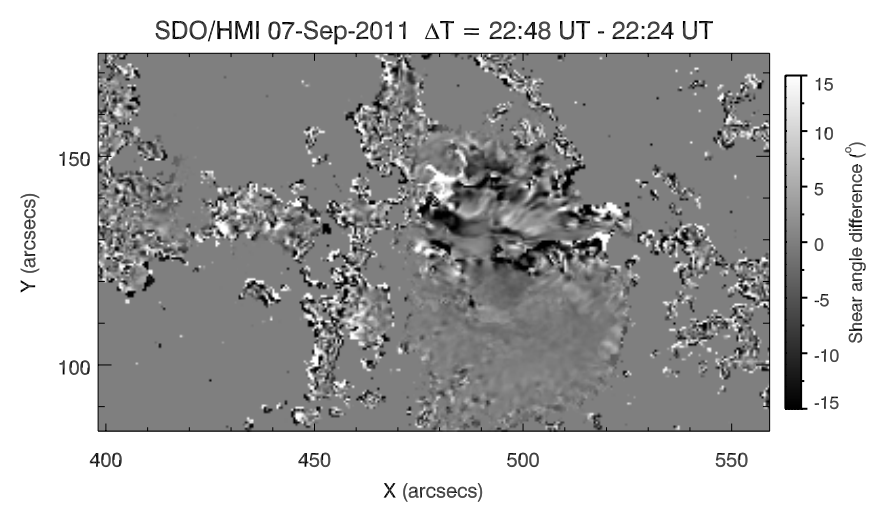

(e)

Fig. 8. Same as Fig. 6, but for the third flare listed in Table 1 .

result cannot exclude the interactions of systems characterized by opposite signs of magnetic helicity flux as being responsible for the solar eruptions (Chandra et al. 2010; Romano et al. 2011).

\subsection{Poynting fluxes}

We corrected the velocities computed by DAVE4VM, hereafter denoted $\boldsymbol{V}$, by removing the plasma flows aligned with the magnetic field, $\boldsymbol{B}$ and considering only the velocities perpendicular to the magnetic field lines, $\boldsymbol{V}_{\perp}$, (Liu \& Schuck 2012):

$\boldsymbol{V}_{\perp}=\boldsymbol{V}-\frac{\boldsymbol{V} \cdot \boldsymbol{B}}{B^{2}} \boldsymbol{B}$

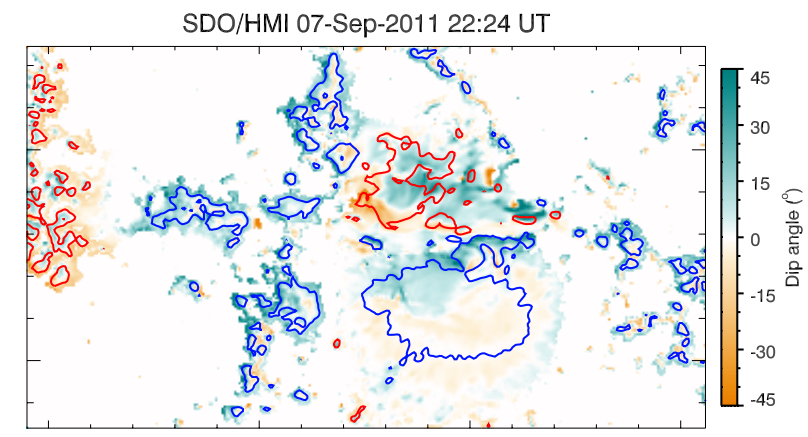

(b)

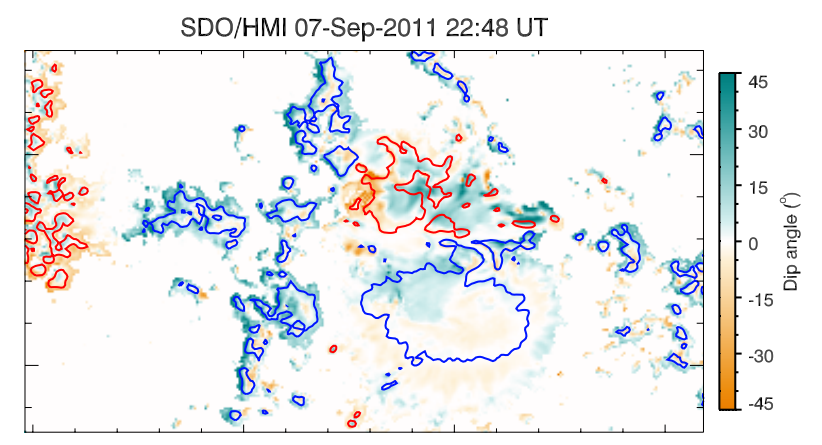

(d)

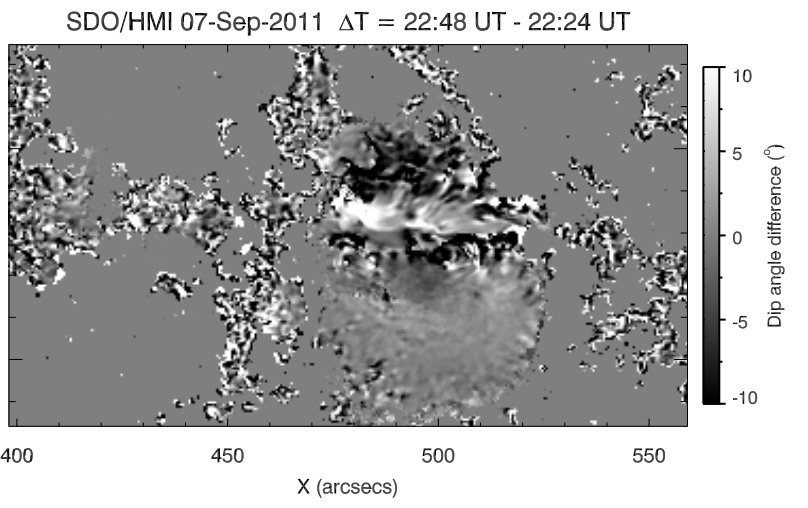

(f)
Then we computed the magnetic energy flux (Poynting flux) across the photosphere, $S$, according to Kusano et al. (2002),

$\frac{\mathrm{d} E}{\mathrm{~d} t}=\frac{1}{4 \pi} \int_{S} B_{t}^{2} V_{\perp n} \mathrm{~d} S+\frac{1}{4 \pi} \int_{S}\left(\boldsymbol{B}_{t} \cdot \boldsymbol{V}_{\perp t}\right) B_{n} \mathrm{~d} S$,

where $\boldsymbol{B}_{t}$ and $B_{n}$ denote the tangential and vertical magnetic fields, and $\boldsymbol{V}_{\perp t}$ and $V_{\perp n}$ are the tangential and vertical components of velocity $\boldsymbol{V}_{\perp}$. In particular, we computed the two terms separately in order to compare the energy flux coming from the emergence of twisted magnetic flux tubes (first term) and from the shearing motions of the magnetic field lines (second term). We show in Fig. 12 the temporal profiles of the accumulated energy in the corona from the emergence term (blue line) and the shear term (red line). We note that during our observation time interval the shearing motions appear to be the main source of magnetic energy in the corona until a few hours before the third 


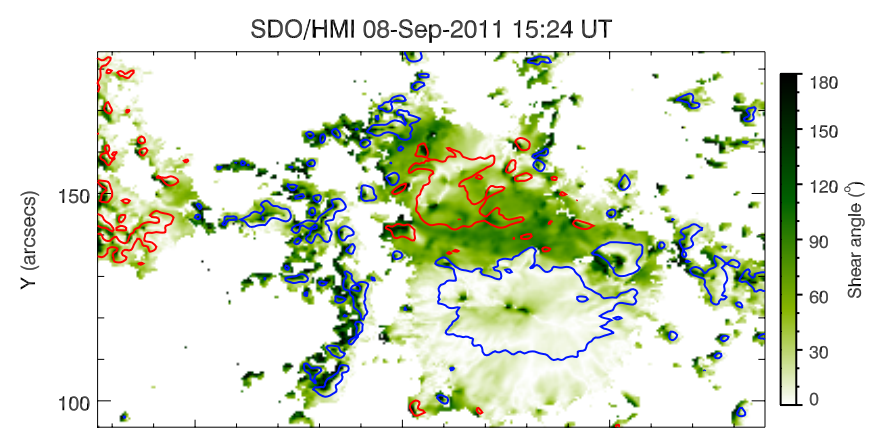

(a)

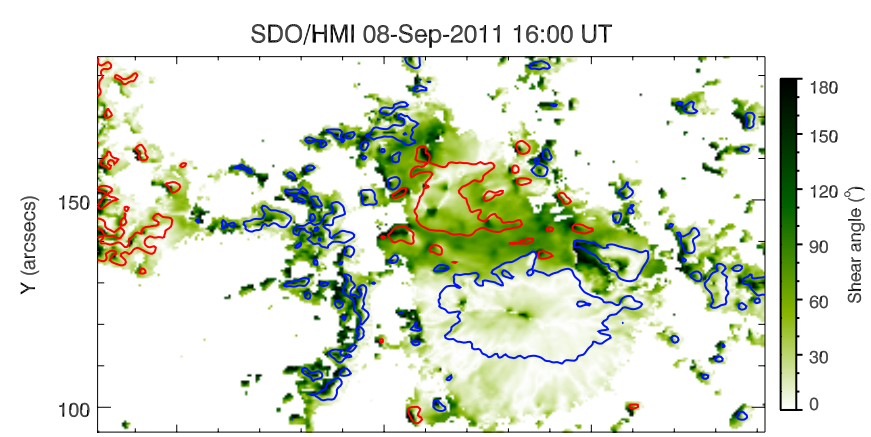

(c)

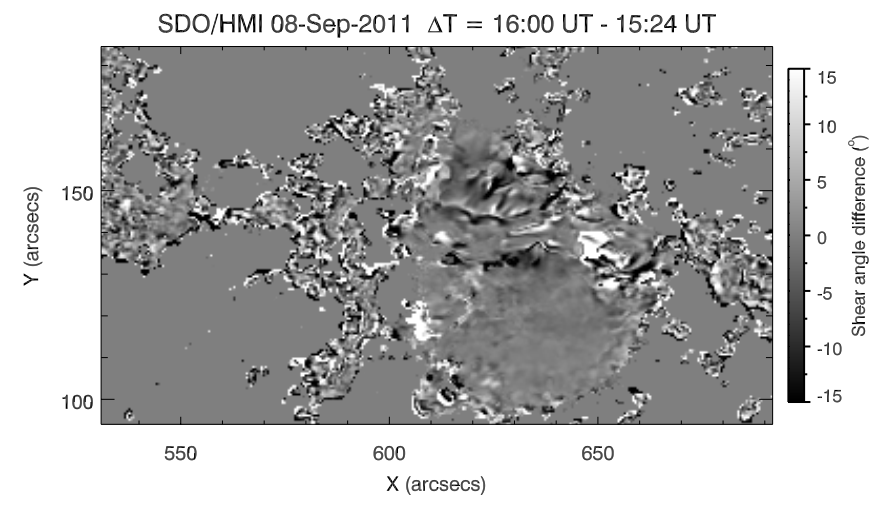

(e)

Fig. 9. Same as Fig. 6, but for the fourth flare listed in Table 1.

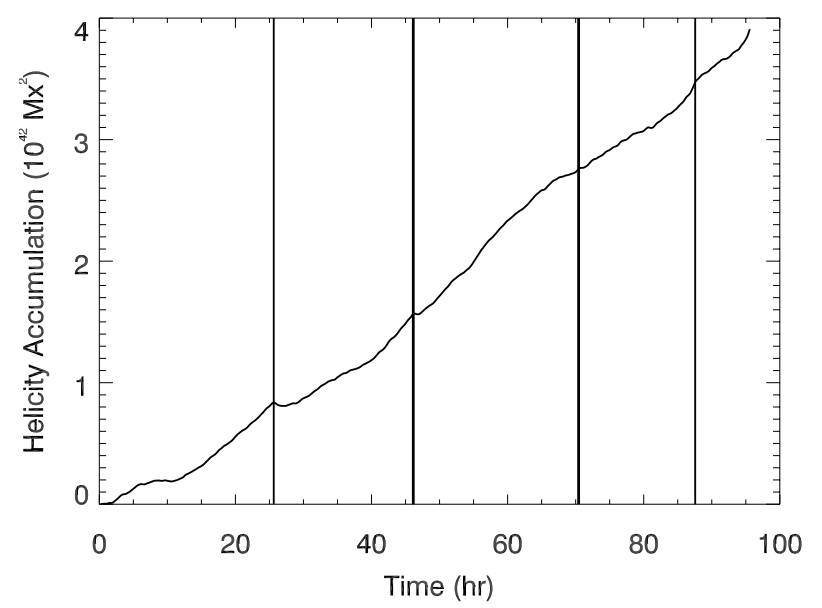

Fig. 10. Accumulation of the magnetic helicity flux in AR NOAA 11283. $t=0$ corresponds to 2011 Sept. 5 at 00:00 UT. The vertical lines indicate the time when the $\mathrm{M}$ and $\mathrm{X}$ flares/CMEs occurred.

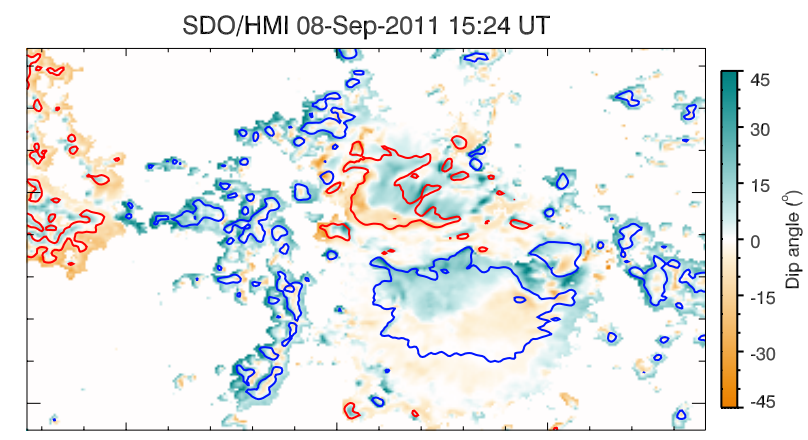

(b)

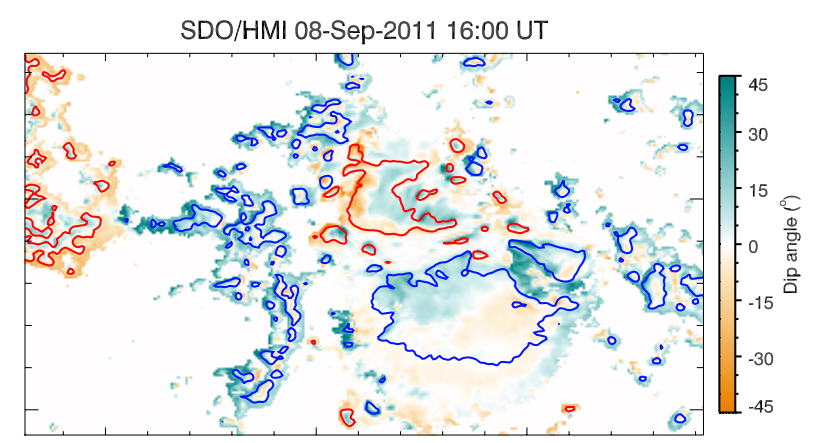

(d)

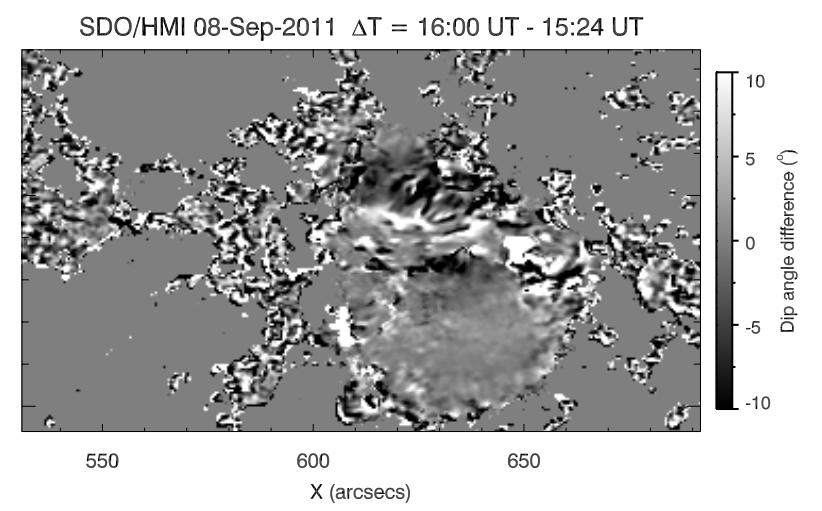

(f)

flare. During the last $20 \mathrm{~h}$ no further energy is accumulated in the corona.

\section{Discussion and conclusions}

The recurrent flares observed in AR 11283 provided an interesting observational tool with which to study the mechanisms at the base of homologous flares. The analysis of the vertical component of the magnetic field observed by HMI/SDO showed some important aspects of the photospheric evolution of the AR during its passage over the solar disk. On the one hand, we did not observe any significant emergence of magnetic field during the period of recurrent flare occurrence, while on the other hand, we detected a peculiar horizontal velocity pattern during our observation time interval. As did Ruan et al. (2014), before the X2.1 class flare we noted a clockwise rotation of the main negative sunspot, but we also found a persistent shear motion of the positive and negative polarities along the PIL. However, from the Poynting fluxes computed from the emergence of the magnetic flux and from the shearing of magnetic field lines due 


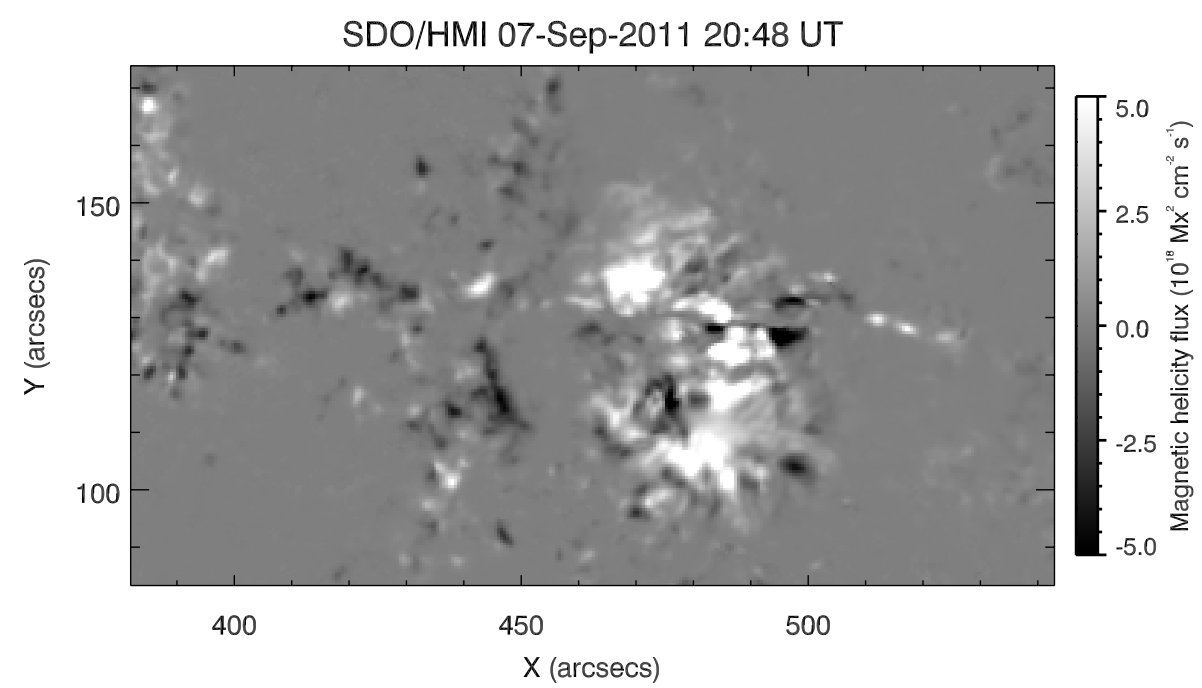

Fig. 11. Helicity flux density map computed with the method of Pariat et al. (2005). Black and white correspond to negative and positive helicity flux density, respectively. The saturation levels are $\pm 5.0 \times 10^{18} \mathrm{Mx}^{2} \mathrm{~cm}^{-2} \mathrm{~s}^{-1}$.

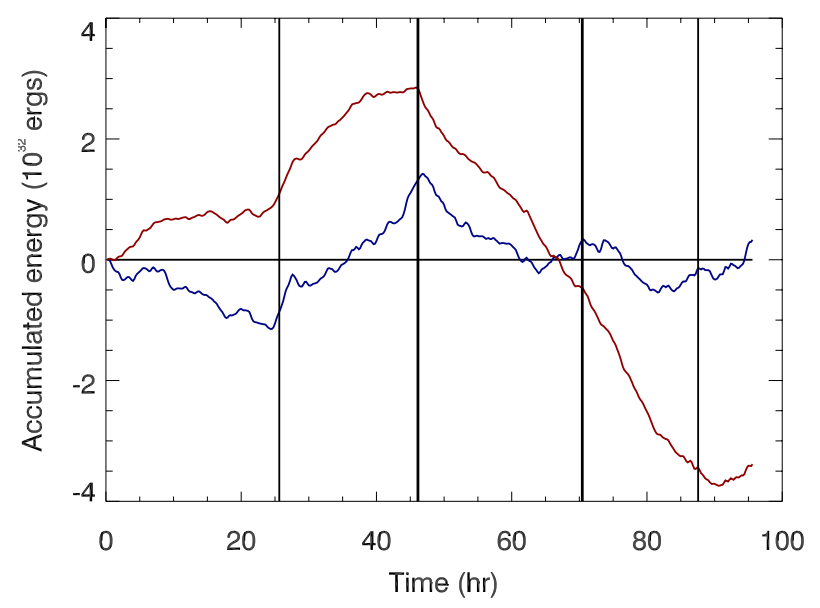

Fig. 12. Temporal profiles of the accumulated energy in the corona from the shear term (red) and emergence term (blue).

to tangential motions on the surface, we found that, even if the energy flux coming from the shearing motions seems to be the main source of energy in the corona during our observation time interval, this energy is lower than the energy released by the M and $\mathrm{X}$ flares. Therefore, it is possible that the flares are also powered by the energy initially present in the magnetic field, while the shearing motions could trigger its release. Nevertheless, we cannot exclude that the uncertainties in measurements of the velocity fields using the DAVE4VM algorithm may lead to an underestimation of the true Poynting fluxes.

These horizontal displacements are probably the main source of the high values of the shear angle near the PIL (about $100^{\circ}$ ). We also note the high magnetic shear and dip angle decrease after each event, especially along the PIL, as shown in the difference maps (see the dark regions in the bottom line of Figs. 6-9). We relate this decrease to the fact that the free energy is stored in non-potential magnetic loops that are stretched upwards and that the free-energy released during the flare must be accompanied by a sudden shrinkage or implosion in the field, as Hudson (2000) conjectured. Therefore, after the flare, the field should become more horizontal, especially above the PIL (Hudson et al. 2008). Moreover, from the comparison of the difference maps over the four events, it seems that the more intense the flare is, the greater the dip and shear variations are. We interpret this effect as a consequence of the different amounts of magnetic flux involved in the magnetic reconfiguration after each flare.

We also note that the differences in the dip angle are more spatially coherent and stronger than the variations in the shear angle across the four events. In particular all the maps of the difference in dip angle show a strong positive core along the PIL and two negative wings away from the PIL. The fact that the dip angle maintained its correlation with the shear angle after each event implies that the flares/CMEs were not able to remove all of the stored free energy and, hence, to significantly modify the magnetic topology of the AR.

The observed motions of the two sunspots of opposite polarities were also responsible for the injection of positive magnetic helicity flux near the PIL. However, taking into account the 3D numerical simulation of Manchester et al. (2004) on the emergence of a magnetic flux rope from the convection zone and its eruption, we cannot exclude that the observed shearing flows are correlated with the previous emergence phase of the AR.

We therefore conclude that the continuous horizontal displacements that contribute to the monotonic injection of magnetic helicity in the corona, coupled with the high shear and dip angles along the main PIL, could result in a configuration where subsequent episodes of loss of equilibrium occurred, leading to recurrent energy release processes, observed as recurrent flares and CMEs.

Acknowledgements. The authors wish to thank the referee for his/her very useful comments and suggestions, which led to a sounder version of the article. This research work has received funding from the European Commissions Seventh Framework Programme under the grant agreements No. 284461 (eHEROES project), No. 312495 (SOLARNET project), No. 606862 (F-Chroma project). This research work is partly supported by the Italian MIUR-PRIN grant 2012P2HRCR on The active Sun and its effects on Space and Earth climate and by Space Weather Italian COmmunity (SWICO) Research Program. The research by the KSU astronomy unit - A.E. and A.S.K. - was supported by King Saud University, Deanship of Scientific Research, College of Science Research Center.

\section{References}

Archontis, V., Hood, A. W., \& Tsinganos, K. 2014, ApJ, 778, 42

Chae, J. 2001, ApJ, 560, L9

Chandra, R., Pariat, E., Schmieder, B., Mandrini, C. H., \& Uddin, W. 2010, Sol. Phys., 261, 127 
P. Romano et al.: Recurrent flares in active region NOAA 11283

DeVore, C. R. \& Antiochos, S. K. 2008, ApJ, 680, 740

Fan, Y., Zweibel, E. G., \& Lantz, S. R. 1998, ApJ, 493, 480

Hoeksema, J. T., Liu, Y., Hayashi, K., et al. 2014, Sol. Phys., 289, 3483

Hudson, H. S. 2000, ApJ, 531, L75

Hudson, H. S., Fisher, G. H., \& Welsch, B. T. 2008, ASP Conf. Ser., 383, 221

Jiang, C., Feng, X., Wu, S. T., Hu, Q. 2013, ApJ, 771, L30

Jiang, C., Wu, S. T., Feng, X., \& Hu, Q. 2014, ApJ, 780, 55

Kliem, B., \& Török, T. 2006, Phys. Rev. Lett., 96, 255002

Kusano, K., Maeshiro, T., Yokoyama, T., \& Sakurai, T. 2002, ApJ, 577, 501

Kusano, K., Bamba, Y., Yamamoto, T. T., et al. 2012, ApJ, 760, 31

Lemen, J. R., Title, A. M., Akin, D. J., et al. 2012, Sol. Phys., 275, 17

Lin, J., Soon, W., \& Baliunas, S. L. 2003, New Astron. Rev., 47, 53

Liu, Y., \& Schuck, P. W. 2012, ApJ, 761, 105
Liu, Y., Hoeksema, J. T., Bobra, M., et al. 2014, ApJ, 785, 13

Magara, T. 2006, ApJ, 653, 1499

Manchester, W., IV, Gombosi, T., DeZeeuw, D. \& Fan, Y. 2004, 610, 588

McClymont, A. N., \& Fisher, G. H. 1989, GMS, 54, 219

Moore, R. L., Sterling, A. C., Hudson, H. S., \& Lemen, J. R. 2001, ApJ, 552, 833

Nitta, N. V., \& Hudson, H. S. 2001, Geophys. Res. Lett., 28, 3801

Pariat, E., Démoulin, P., \& Berger, M. A. 2005, A\&A, 439, 1191

Romano, P., \& Zuccarello, F. 2011, A\&A, 535, A1

Ruan, G., Chen, Y., Wang A., et al. 2014, ApJ, 784, 165

Schou, J., Scherrer, P. H., Bush, R. I., et al. 2012, Sol. Phys., 275, 229

Schuck, P. W. 2008, ApJ, 683, 1134

Shibata, K., \& Magara, T. 2011, Liv. Rev. Sol. Phys., 8, 6 